\title{
Evaluation of Diagnostic Tests by Evanescence Biosensor Technology for Rapid Phenotyping of the Human Platelet Alloantigens 1a and 5b
}

\author{
Yves Merieux $^{a} \quad$ Celestine Schwab ${ }^{b} \quad$ Maurine Saint-Cyr ${ }^{a} \quad$ Gabi Rink $^{b} \quad$ Claudio Rhyner $^{c, d}$ \\ Manfred Schawaller ${ }^{c}$ Peter Bugert ${ }^{b}$ \\ aEtablissement Français du Sang Auvergne-Rhône-Alpes, Lyon, France; \\ ${ }^{b}$ Institute of Transfusion Medicine and Immunology, Heidelberg University, Medical Faculty Mannheim, German Red Cross Blood Service \\ Baden-Württemberg - Hessen, Mannheim, Germany; \\ ${ }^{c}$ Davos Diagnostics, Davos, Switzerland; d Swiss Institute of Asthma and Allergy Research (SIAF), Davos, Switzerland
}

\section{Keywords}

HPA phenotyping · Genotyping · Neonatal alloimmune thrombocytopenia

\begin{abstract}
Background: The human platelet alloantigens (HPA) HPA-1a and HPA-5b are located on glycoproteins on the platelet surface and are the most relevant to cause neonatal alloimmune thrombocytopenia (NAIT). The antigens are defined by single nucleotide polymorphisms (SNPs) in the glycoprotein genes, and the antigen status can be determined by genotyping the SNPs. However, genotyping is time-consuming and costly depending on the method and sample throughput. Here, we tested the reliability of the evanescence wave based fluorescence (EVA) biosensor technology for the rapid phenotyping of the HPA-1a and HPA-5b antigens on blood donor samples in two laboratories. Methods: HPA-1a and HPA-5b phenotyping was performed on EDTA blood samples from 336 blood donors (Lyon: 216 donors; Mannheim: 120 donors) using EVA typing assays and the biosensor system (Davos Diagnostics, Davos, Switzerland). For genotyping, validated PCR-SSP and TaqMan-PCR methods were used. Results: HPA-1a phenotyping was positive for all samples with HPA-1aa ( $n=244$; EVA value 807 $\pm 167 \mathrm{U} / \mathrm{s}$ ) and HPA-1ab ( $\mathrm{n}=82 ; 542 \pm 110 \mathrm{U} / \mathrm{s}$ ) genotypes. All samples $(n=10)$ with negative EVA values $(<10$ $\mathrm{U} / \mathrm{s}$ ) had the HPA-1bb genotype. HPA-5b phenotyping was negative for all HPA-5aa genotypes $(n=267)$ and positive for the HPA-5ab ( $n=66 ; 83 \pm 22 \mathrm{U} / \mathrm{s}$ ) and HPA-
\end{abstract}

$5 b b(n=3 ; 118 \pm 25 \mathrm{U} / \mathrm{s}$ ) genotypes. EVA values from heterozygotes were significantly lower compared to HPA-1a or HPA-5b homozygotes. A strong correlation of the EVA values with the platelet count in the blood samples was observed. Conclusion: EVA is a reliable method for rapid phenotyping of the clinically relevant HPA-1a and HPA-5b platelet antigens. All phenotyping results were $100 \%$ concordant with the HPA- 1 or HPA-5 genotype. The test can be performed from only $10 \mu \mathrm{l}$ of fresh or frozen blood samples within less than 15 min time-toresult.

(c) 2018 S. Karger AG, Basel

\section{Introduction}

The human platelet alloantigens (HPA) are located on glycoproteins on the platelet surface and can induce alloimmune reactions as a common cause of neonatal alloimmune thrombocytopenia (NAIT), posttransfusion purpura, or refractoriness after platelet transfusion [1-3]. The HPA-1a and HPA-5b antigens are the most relevant antigens in more than $90 \%$ of NAIT cases or transfused patients [4]. The knowledge about the antigen status of a patient or a donor is important for the decision regarding further treatment [5]. The HPA-1 and HPA-5 antigens are defined by single nucleotide polymorphisms (SNPs) in the glycoprotein genes. The antigen status is mostly done by genotyping the corresponding SNPs, and numerous methods have been developed over the years (for review see [6]). However, genotyping is time-consuming and costly depending on the method and sample throughput.

\section{KARGER}

(C) 2018 S. Karger AG, Basel 
Immunosorbent assays, like enzyme linked immunosorbent assay (ELISA) are the most commonly used method for the highly specific and highly sensitive detection of biological analytes in research and in vitro diagnostics (IVD) [7]. However, due to long incubation times and several periods of hands-on time and multiple washing steps ELISA take hours to achieve a result. The evanescence wave-based fluorescence (EVA) biosensor technology combines high specificity and sensitivity with a short time-to-result of only $12 \mathrm{~min}[8,9]$. In this immunoassay with one single pipetting step and avoiding any washing step the specific binding of the analyte to the surface of a biosensor chip is monitored in real time.

In this study we tested the reliability of the EVA technology for rapid phenotyping of the HPA-1a and HPA-5b antigens in healthy blood donors. The results from phenotyping were compared to genotyping data of the donors. All measurements were performed independently in two laboratories (Lyon, France and Mannheim, Germany) and revealed comparable data.

\section{Material and Methods}

\section{Blood Samples}

For HPA-1a and HPA-5b phenotyping and genotyping we used EDTA blood samples from 216 blood donors (freshly collected or stored at $4{ }^{\circ} \mathrm{C}$ ) at the Etablissement Français du Sang (EFS) Auvergne-Rhône-Alpes, Lyon, and from 120 blood donors (stored $-30^{\circ} \mathrm{C}$ ) at the Institute of Transfusion Medicine and Immunology, Mannheim. All donors gave their written consent to use blood samples for research and test validation.

In order to test the limitations of the EVA technology with regard to platelet numbers in the blood samples we generated artificial thrombocytopenic blood from healthy donors heterozygous for HPA-1ab or HPA-5ab. Briefly, after centrifugation of EDTA blood samples for $15 \mathrm{~min}$ at $150 \times g$ the platelet-rich plasma (PRP) was removed. An aliquot of PRP was kept for later titration of platelets, and the rest of PRP was centrifuged for $10 \mathrm{~min}$ at 5,000 $\times g$ to obtain platelet-poor plasma (PPP). Each EDTA blood sample was mixed with increasing amounts of PRP and decreasing amounts of PPP so that we obtained 5 samples of equal volume with different platelet counts from each donor. The platelet counts in the blood samples were determined by using hematology analyzer (CELL-DYN Ruby; Abbot Core Laboratory, Wiesbaden, Germany).

\section{HPA-1 $a$ and HPA-5b Phenotyping Using EVA Technology}

The EDTA blood samples were directly subjected to EVA phenotyping of the HPA-1a and HPA-5b antigens by using the biosensor system (Davos Diagnostics, Davos, Switzerland). The antigen typing assays are sandwich immunoassays that distinguish between antigen-positive and -negative platelets. The 8 wells of an EVA chip are coated with an anti-glycoprotein antibody (GPIIbIIIa for HPA-1 or GPIaIIa for HPA-5). A fluorophore-conjugated (allophycocyanin; APC) anti-HPA-1a (Davos Diagnostics) or anti-HPA-5b (EFS Lyon) antibody and all reagents for the test are pre-aliquoted and dried in the wells. When a blood sample mixed with lysis buffer is added to the well the dried reagents dissolve and the binding reaction starts immediately. The biosensor principle is based on the real-time measurement of the binding reaction in the evanescent field which is generated at the bottom $200 \mathrm{~nm}$ of each well by total reflection of the excitation laser light (fig. 1). During the 10-min continuous measurement the fluorescent-labeled antibodies specifically bind to antigen-positive platelets that also bind to the surface of the well. The increase in fluorescent signal over time is recorded as slope (U/s) by the EVA reader and correlates with the concentration of the antigen in the sample (fig. 1). Limitations of the test are given by the number of platelets in the blood sample.

The HPA-1a and HPA-5b typing assays were used according to the standard protocol. Briefly, $20 \mu \mathrm{l}$ anti-coagulated EDTA blood was mixed with $40 \mu \mathrm{l}$ lysis buffer (included in the typing assay kit) and vortexed for a few seconds; then $20 \mu$ lof the lysate was added to each of two wells of the EVA chip. Thus, 4 samples were measured in duplicates on one EVA chip (8 wells). Typing of both antigens required $40 \mu \mathrm{l}$ EDTA blood and two different EVA chips. The blood lysate dissolves the detection reagent which is already in dried form deposited in the edges of the wells. The necessary test information is presented to the reader via a $2 \mathrm{D}$ barcode and specifies the test parameters and the way of interpretation in a batch-specific manner. The eight samples on the EVA chip are measured simultaneously for the bound fluorophores in the evanescent filed over $10 \mathrm{~min}$ time, and the result is given as SLOPE in U/s. A positive result is the linear increase of emitted light with a slope of $>30 \mathrm{U} / \mathrm{s}$, negative samples show a background signal of $\leq 10 \mathrm{U} / \mathrm{s}$. Slopes between 10-30 U/s indicate intermediate results, and the measurement should be repeated. Each sample was measured twice for each antigen, and all values were exported to MS Excel (Microsoft Office 2010; Microsoft Corp., Redmond, WA, USA) and SPSS (Version 12.0; SPSS Inc., Chicago, IL, USA) for further calculations. Significance ( $\mathrm{p}<$ 0.05 ) of differences in EVA values between the genotype groups were calculated by using Student's t-test (unpaired, two-sided). The correlation of platelet counts with EVA values was calculated by Pearson correlation analysis.

For quality control of the EVA reader, a control kit (R6 Control Kit; Davos Diagnostics) was used which is based on avidin-binding of a biotinylated fluorophore. The control chip is coated with avidin, and the control conjugate contains biotin conjugated to fluorophore (Cy5). The control chip is designed to create a negative and a positive signal by adding the control conjugate.

\section{HPA-1 and HPA-5 Genotyping}

EFS, Lyon: DNA was isolated from EDTA-anticoagulated blood using a commercial system (Maxwell 16 Blood DNA purification system; Promega, Fitchburg, WI, USA). For HPA-1 and HPA-5 genotyping, in-house PCR-SSP methods were performed in Perkin-Elmer PE 9700 thermal cycler. The amplification results were read on a $2 \%$ agarose gel with ethidium bromide. The HPA- 1 and HPA- 5 genotypes could be determined by the presence or absence of PCR products of the expected size [10].

Institute of Transfusion Medicine and Immunology, Mannheim: DNA was isolated from EDTA-anticoagulated blood using a commercial system (QIAamp Blood DNA Mini Kit; Qiagen, Hilden, Germany). For HPA-1 and HPA-5 genotyping, validated TaqMan-PCR assays (HPA-1: assay ID C___818008_30 for rs5918; HPA-5: assay ID C_27862812_10 for rs1801106; Applied Biosystems, Darmstadt, Germany) were used according to the standard protocol of the manufacturer. The FAM and VIC fluorescence was measured after PCR (end-point detection) and indicated homozygous or heterozygous HPA-1 and HPA-5 genotypes of the samples [11].

\section{Results}

\section{HPA-1a Phenotyping and HPA-1 Genotyping}

HPA-1a phenotyping was performed on 336 blood donor samples from the two institutes by using the EVA technology. Each sample was tested twice, and the results were always concordant. The mean variation between the two measurements was $11.4 \%$ (range $0.1-57.8 \%$ ) for the HPA-1a-positive samples. Phenotyping revealed a positive result for 326 samples (97\%) and a negative result for 10 samples (3\%). This was in complete concordance to genotyping, i.e. all EVA HPA-1a-positive samples were positive for the HPA-1a allele, either homozygous (HPA-1aa; $\mathrm{n}=244$ ) or heterozygous (HPA-1ab; $\mathrm{n}=82$ ). All EVA HPA-1a-negative samples had the HPA-1bb $(n=10)$ genotype. The EVA values of HPA-1aa genotypes were significantly higher than HPA-1ab genotypes (807 $\pm 167 \mathrm{U} / \mathrm{s}$ vs. $542 \pm 110 \mathrm{U} / \mathrm{s} ; \mathrm{p}<0.001$ ) but, as expected, HPA-1a phenotyping could not discriminate between the two genotypes 




Fig. 1. Description of the EVA principle. A The exciting light beam is reflected under total internal reflection fluorescence (TIRF) at the liquid-solid interface at the bottom of a well in the biosensor chip. By this optical phenomenon a 200 nanometer bottom layer of the adjacent liquid is illuminated. Only fluorophores localized in this evanescence field will absorb and emit photons. B The measurement is performed in the EVA reader R6. C The EVA chip is a single-use consumable that includes 8 wells coated with an anti-glycoprotein-specific (GPIIbIIIa for HPA-1 or GPIaIIa for HPA-5) antibody. D On the left panel a kinetic reaction of two HPA-la-positive and two negative samples are depicted (each sample in duplicates). On the right panel, the calculated slopes (U/s) of the kinetic reactions are shown.

(fig. 2A). The EVA values for the HPA-1aa samples were significantly higher in the Lyon laboratory compared to the Mannheim laboratory $(833 \pm 169 \mathrm{U} / \mathrm{s}$ vs. $758 \pm 152 \mathrm{U} / \mathrm{s} ; \mathrm{p}=0.001)$. The HPA$1 \mathrm{ab}$ samples gave comparable results $(542 \pm 113$ and $541 \pm 106 \mathrm{U} / \mathrm{s}$, respectively). In both laboratories, HPA-1a phenotyping showed $100 \%$ sensitivity and specificity compared to the HPA-1 genotypes.

Artificial thrombocytopenic samples with increasing numbers of platelets were generated from 3 healthy volunteers with the HPA-1ab genotype. A total of 15 samples with platelet counts between 23 and 240 platelets/nl were measured twice for the HPA-1a antigen. The EVA values significantly correlated $(\mathrm{r}=0.914 ; \mathrm{p}>$ 0.001) with the platelet count in the samples (fig. 2B). All samples showed positive HPA-1a typing with the lowest EVA value of 107.5 $\mathrm{U} / \mathrm{s}$ which is clearly distinct from the intermediate range of 10-30 $\mathrm{U} / \mathrm{s}$.

\section{HPA-5b Phenotyping and HPA-5 Genotyping}

HPA-5b phenotyping was performed on the same 336 blood donor samples by using the EVA technology. Phenotyping revealed a positive result for 69 samples $(20.5 \%)$ and a negative result for 267 samples (79.5\%). Each sample was tested twice, and 

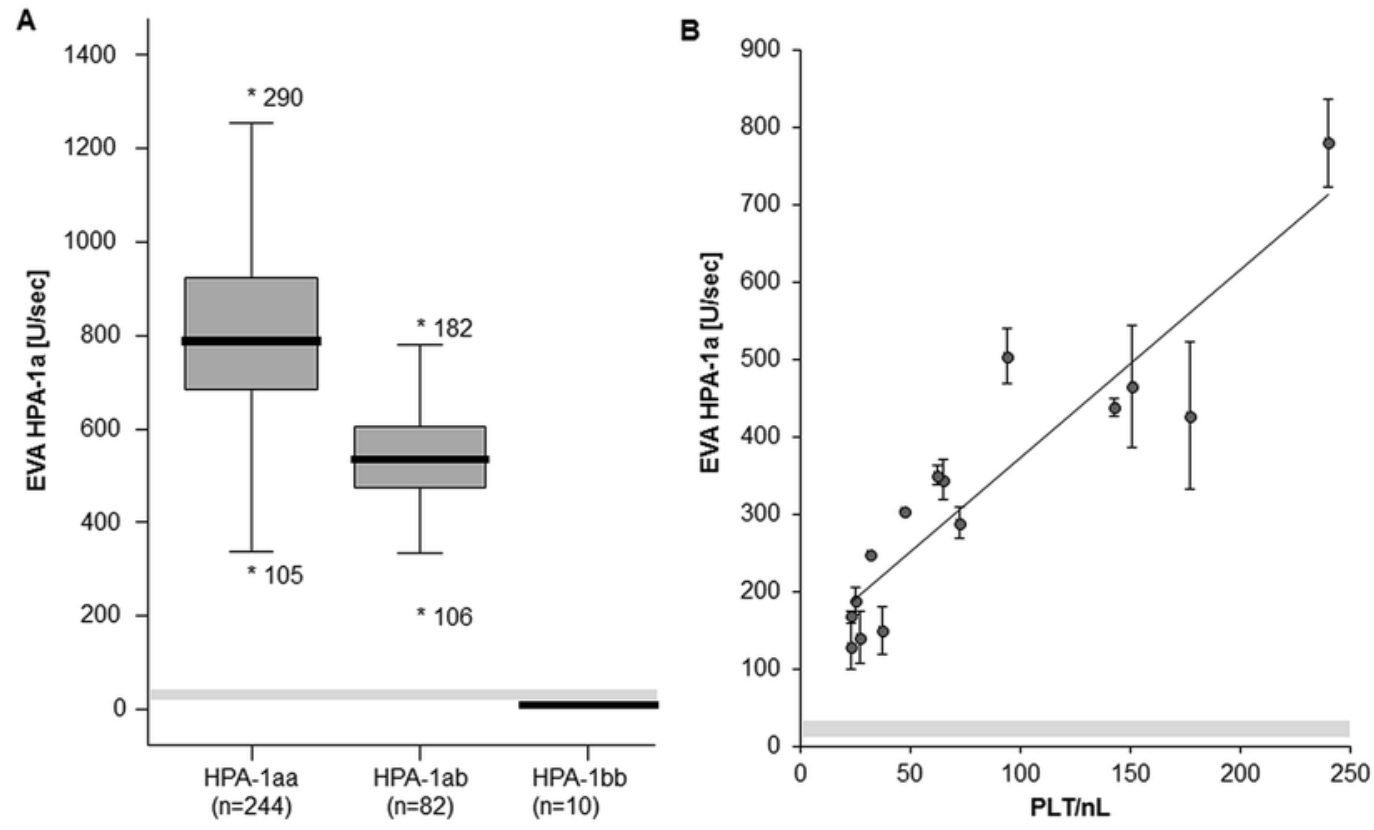

Fig. 2. A Comparison of EVA HPA-1a phenotyping data with the HPA-1 genotype from 336 blood donors. Results from phenotyping are given in arbitrary EVA units $(\mathrm{U} / \mathrm{sec})$. Outlier samples are indicated by asterisk $\left(^{*}\right)$ and sample number. B In blood samples from 3 HPA-1ab heterozygous donors platelets were depleted by centrifugation to obtain thrombocytopenic blood. After titration of platelets to the samples the platelet counts were determined and the HPA-1a antigen was measured by EVA in duplicates. The platelet numbers significantly correlated with the EVA values $(r=0.914 ; \mathrm{p}<0.001)$. Even at very low platelet counts of $<30$ platelets/nl a clearly positive EVA result was observed. The gray-shaded area indicates the range of intermediate values of 10-30 U/s.

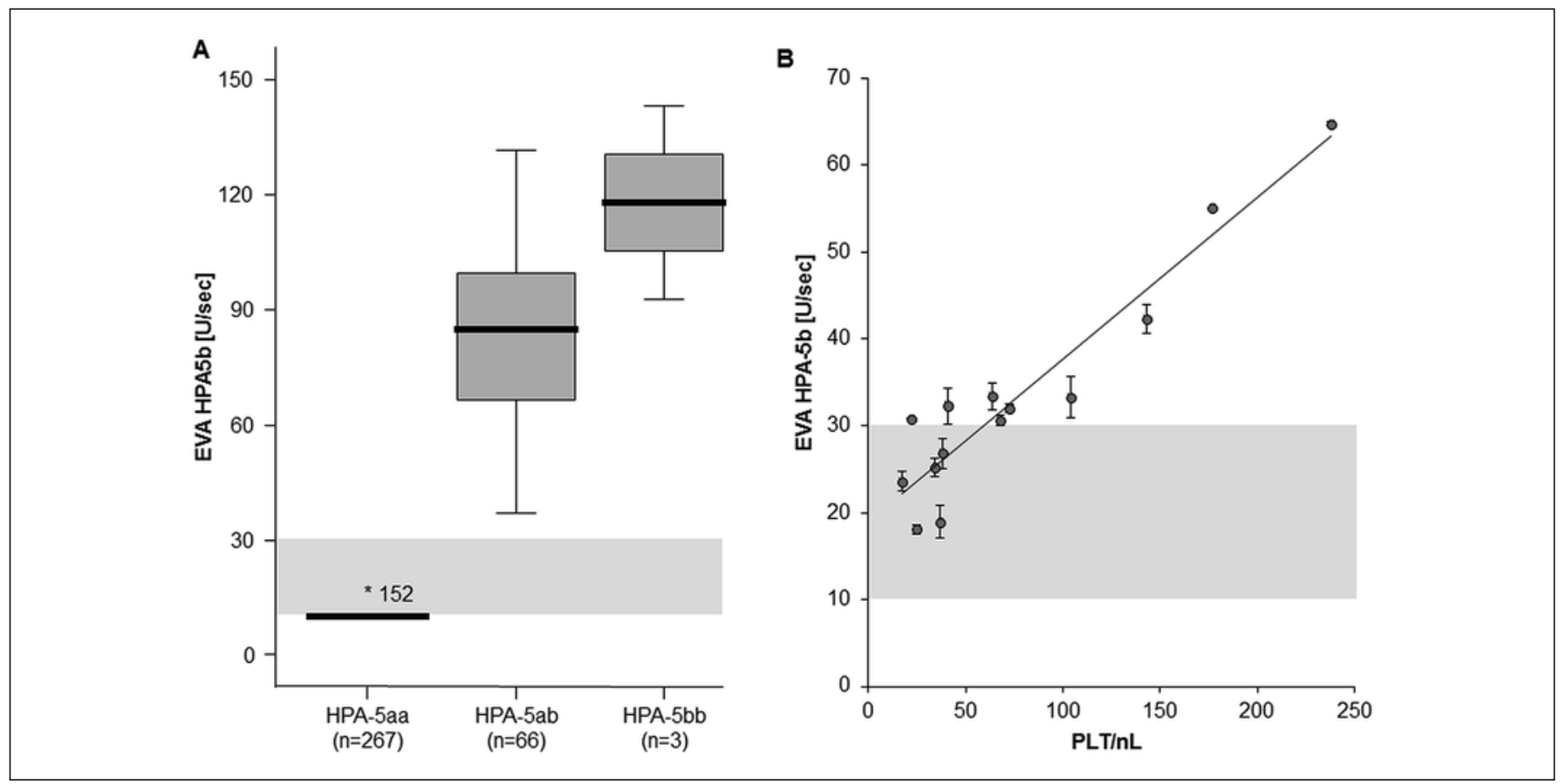

Fig. 3. A Comparison of EVA HPA-5b phenotyping data with the HPA-5 genotype from 336 blood donors. Results from phenotyping are given in arbitrary EVA units $(\mathrm{U} / \mathrm{sec})$. Outlier samples are indicated by asterisk $\left(^{*}\right)$ and sample number. B In blood samples from 3 HPA-5ab heterozygous donors platelets were depleted by centrifugation to obtain thrombocytopenic blood. After titration of platelets to the samples the platelet counts were determined and the HPA-5b antigen was measured by EVA in duplicates. The platelet numbers significantly correlated with the EVA values $(\mathrm{r}=0.949 ; \mathrm{p}<0.001)$. With platelet counts lower than 50 platelets/nl the EVA result was in the intermediate range (10-30 U/s; gray-shaded area) in 5 of 7 samples. None of the samples revealed a negative EVA result ( $<10 \mathrm{U} / \mathrm{s})$. 
the results were always concordant. The mean variation between the two measurements was $8.2 \%$ (range $0.1-50.4 \%$ ) for the HPA-5b-positive samples. All HPA-5b-negative samples with EVA values $\leq 10$ had the HPA-5aa genotype. In one HPA-5aa sample (sample 152 in fig. $3 \mathrm{~A}$ ), one EVA value was negative $(<10$ $\mathrm{U} / \mathrm{s}$ ) and the other was $23 \mathrm{U} / \mathrm{s}$, which is in the intermediate range (10-30 U/s). However, the mean value of $17 \mathrm{U} / \mathrm{s}$ was clearly distinct from the lowest mean value ( $37 \mathrm{U} / \mathrm{s}$ ) of the HPA-5b-positive samples. All EVA HPA-5b-positive samples were positive for the HPA-5b allele, either heterozygous (HPA-5ab; $n=66$ ) or homozygous (HPA-5bb; $\mathrm{n}=3$ ). The EVA values of HPA-5bb genotypes were significantly higher than those of HPA-5ab genotypes ( $83 \pm$ $22 \mathrm{U} / \mathrm{s}$ vs. $118 \pm 25 \mathrm{U} / \mathrm{s} ; \mathrm{p}=0.011$ ) but, as expected, the HPA-5b phenotyping could not discriminate between assay homo- and heterogeneous donors (fig. 3A). For the HPA-5ab samples, the EVA values were significantly higher in the Lyon laboratory compared to the Mannheim laboratory $(98 \pm 49 \mathrm{U} / \mathrm{s}$ vs. $64 \pm 15 \mathrm{U} / \mathrm{s} ; \mathrm{p}$ $=0.004$ ). In both laboratories, HPA-5b phenotyping showed $100 \%$ sensitivity and specificity compared to the HPA-5 genotypes. The 3 samples with HPA-5bb genotype were analyzed only in the Mannheim laboratory.

Artificial thrombocytopenic samples with increasing numbers of platelets were generated from 3 healthy volunteers with the HPA-5ab genotype. A total of 14 samples with platelet counts between 17 and 238 platelets/nl were measured twice for the HPA-5b antigen. The EVA values significantly correlated $(r=0.949 ; \mathrm{p}>$ 0.001 ) with the platelet count in the samples (fig. 3B). In 5 of the 7 samples with platelet counts $<50$ platelets/nl, the EVA values were in the intermediate range $(10-30 \mathrm{U} / \mathrm{s})$. None of the samples was negative, and the lowest EVA value was $17.6 \mathrm{U} / \mathrm{s}$.

\section{Discussion}

EVA is a reliable method for rapid phenotyping of the clinically relevant HPA-1a and HPA-5b platelet antigens. Interestingly, storage time and temperature of the blood samples significantly affected the EVA values, i.e. fresh or non-frozen samples stored for some days (Lyon laboratory) gave significantly higher values than samples that have been stored frozen for up to 2 years (Mannheim laboratory). However, the qualitative phenotyping results (antigenpositive or -negative) were not affected. All samples were measured twice with mean variation between the two measurements of $11.4 \%$ for HPA-1a and $8.2 \%$ for HPA-5b. All phenotyping results were $100 \%$ concordant with the HPA- 1 or HPA- 5 genotype of the tested samples.

The impact of platelet-specific auto- or alloantibodies in samples from immunized patients on EVA-based antigen typing is unknown. High-titer anti-HPA-1a or anti-HPA-5b antibodies may interfere with the EVA typing. Further limitations have to be considered with very low platelet counts in the blood samples. As shown by testing artificial thrombocytopenic blood samples, the EVA values significantly correlated with platelet counts, and the sensitivity of the HPA-1a test was much higher than that of the
HPA-5b test. This may be due to a different antibody affinity and to different copy numbers of the glycoproteins on platelets. For HPA-1a typing, a clearly positive result was achieved even with less than 30 platelets/nl. The EVA values in the HPA-5b test were generally lower compared to the HPA-1a test. With platelet counts $<50$ platelets/nl, the EVA values for HPA-5b were often in the intermediate range of 10-30 U/s. However, none of the HPA-5b samples was tested false-negative, even with platelet counts of $<20$ platelets/nl.

The frequencies of positive and negative phenotypes for HPA$1 \mathrm{a}$ and HPA-5b found by the EVA technology corresponds to what has been reported for HPA by using other immunophenotyping methods (Ref. [12]: HPA-1a negative 2.4\%; HPA-5b negative 81.4\%). Phenotyping HPA-1a and HPA-5b in parallel to molecular typing allows to dedicate mutations that could affect PCR assays and which generates false-negative results as reported before [13-15].

The monoclonal antibody-specific immobilization of platelet antigens (MAIPA) technique is used in specialized laboratories for the identification of platelet-specific antibodies [16]. MAIPA methods can also be applied for HPA phenotyping by using human serum containing specific HPA antibodies or by using monoclonal antibodies to certain HPA such as HPA-1a and HPA-5b. However, MAIPA is time-consuming $(>5 \mathrm{~h})$, and about $2 \times 10^{6}$ platelets are required for determination of each antigen. The antigen-specific capture assay (ASCA) was reported for HPA-1a phenotyping and showed complete concordance with the genotypes of 139 blood donors [17]. The ASCA is much faster compared to ELISA or MAIPA but also includes several pipetting steps and centrifugation.

The EVA technology enables typing of donors for (currently) HPA-1a and HPA-5b in a fast manner (12 min per antigen) without prior sample preparation and with only $10 \mu$ blood sample per test. For phenotyping of patients and blood donors, the EVA antigen-specific assays and the EVA reader system including software are available as IVD products (Davos Diagnostics). It could be implemented to routine diagnosis in order to provide platelet concentrates for compatible transfusion in immunized patients. The application of the EVA technology could also be discussed with regard to routine screening of pregnant women for the HPA-1a antigen in the surveillance of neonatal thrombocytopenia. However, further automation is not available so far, and the chip format with 8 wells is not designed for high throughput screening.

\section{Disclosure Statement}

Claudio Rhyner and Manfred Schawaller are employed by Davos Diagnostics AG, Switzerland. All other authors declare no conflict of interest. The EVA HPA-1a and HPA-5b chips required for this study were provided by Davos Diagnostics for free.
Merieux/Schwab/Saint-Cyr/Rink/Rhyner/ Schawaller/Bugert 


\section{References}

1 Hayashi T, Hirayama F: Advances in alloimmune thrombocytopenia: perspectives on current concepts of human platelet antigens, antibody detection strategies, and genotyping. Blood Transfus 2015;13:380-390.

2 Pacheco LD, Berkowitz RL, Moise KJ Jr, Bussel JB, McFarland JG, Saade GR: Fetal and neonatal alloimmune thrombocytopenia: a management algorithm based on risk stratification. Obstet Gynecol 2011;118:11571163.

3 Novotny VM: Prevention and management of platelet transfusion refractoriness. Vox Sang 1999;76:1-13.

4 Kiefel V, König C, Kroll H, Santoso S: Platelet alloantibodies in transfused patients. Transfusion 2001;41: 766-770.

5 Juskewitch JE, Norgan AP, De Goey SR, Duellman PM, Wakefield LL, Gandhi MJ, Stubbs JR, Kreuter JD: How do I ... manage the platelet transfusion-refractory patient? Transfusion 2017;57:2828-2835.

6 Curtis BR, McFarland JG: Human platelet antigens 2013. Vox Sang 2014;106:93-102.

7 Grange RD, Thompson JP, Lambert DG: Radioimmunoassay, enzyme and non-enzyme-based immunoassays. Br J Anaesth 2014;112:213-216.
8 Trévisan M, Schawaller M, Quapil G, Souteyrand E, Mérieux Y, Cloarec JP: Evanescent wave fluorescence biosensor combined with DNA bio-barcode assay for platelet genotyping. Biosens Bioelectron 2010;26: 1631-1637.

9 Schawaller M, Rhyner C, Wiki M, Quapil G, Zuberbier T, Akdis CA, Crameri R: Evanscence wave-based technology for the rapid and sensitive quantification of biological analytes. J Allergy Clin Immunol 2018;141: 817-820.

10 Mérieux Y, Debost M, Bernaud J, Raffin A, Meyer F, Rigal D: Human platelet antigen frequencies of platelet donors in the French population determined by polymerase chain reaction with sequence-specific primers. Pathol Biol (Paris) 1997;45:697-700.

11 Bugert P, McBride S, Smith G, Dugrillon A, Klüter H, Ouwehand WH, Metcalfe P: Microarray-based genotyping for blood groups: comparison of gene array and $5^{\prime}$-nuclease assay techniques with human platelet antigen as a model. Transfusion 2005;45:654-659.

12 Simsek S, Faber NM, Bleeker PM, Vlekke AB, Huiskes E, Goldschmeding R, von dem Borne AE: Determination of human platelet antigen frequencies in the Dutch population by immunophenotyping and DNA (allele-specific restriction enzyme) analysis. Blood 1993;81:835-840.
13 Bertrand G, Bianchi F, Chenet C, Martageix C, Blanchet P, Bäumler M, Kaplan C: New mutation in the platelet beta3-integrin gene: implication for the diagnosis of fetomaternal alloimmunization. Transfusion 2006;46:2138-2141.

14 Bertrand G, Kaplan C, Kennel A, Perrier P, Moncharmont P: New mutation in the beta 3-integrin (GPIIIa) gene inducing HPA-1 genotyping discrepancies. Transfusion 2010;50:1589-1591.

15 Bertrand G, Jallu V, Beranger T, Bianchi F, Casale C Dufour V, Chenet C, Quesne J, Martageix C, Kaplan C: HPA-5 typing discrepancy reveals an Ile503Leu substitution in platelet GPIa ( $\alpha 2$ integrin). Vox Sang 2013; 105:73-76.

16 Kiefel V, Santoso S, Weisheit M, Müeller-Eckhardt C: Monoclonal antibody-specific immobilization of platelet antigens (MAIPA): a new tool for the identification of platelet-reactive antibodies. Blood 1987;70:17221726.

17 Meyer O, Agaylan A, Bombard S, Kiesewetter H, Salama A: A novel antigen-specific capture assay for the detection of platelet antibodies and HPA-1a phenotyping. Vox Sang 2006;91:324-330. 